OPEN ACCESS

Edited by:

Angelo Quartarone

University of Messina, Italy

Reviewed by:

Mingrui Xia,

Beijing Normal University, China

Francesco Di Lorenzo,

Santa Lucia Foundation (IRCCS), Italy

*Correspondence:

Dan-tao Peng

dantao.peng@outlook.com

${ }^{t}$ These authors have contributed equally to this work

Received: 18 August 2020

Accepted: 05 February 2021

Published: 10 March 2021

Citation:

Zhou Z, Zhu R, Shao W,

Zhang S-j, Wang L, Ding $X$-j and

Peng D-t (2021) Changes

in Resting-State Functional

Connectivity of Cerebellum

in Amnestic Mild Cognitive

Impairment and Alzheimer's Disease:

A Case-Control Study.

Front. Syst. Neurosci. 15:596221. doi: 10.3389/fnsys.2021.596221

\section{Changes in Resting-State Functional Connectivity of Cerebellum in Amnestic Mild Cognitive Impairment and Alzheimer's Disease: A Case-Control Study}

\author{
Zhi Zhou'th, Rui Zhu't, Wen Shao', Shu-juan Zhang ${ }^{1}$, Lei Wang ${ }^{1}$, Xue-jiao Ding ${ }^{1}$ and \\ Dan-tao Peng ${ }^{1 *}$
}

${ }^{1}$ Department of Neurology, China-Japan Friendship Hospital, Beijing, China, ${ }^{2}$ Department of Neurology, Beijing Geriatric Hospital, Beijing, China

This case-control study is aimed to investigate the correlation of altered functional connectivity $(\mathrm{FC})$ in cerebellum with cognitive impairment in amnestic mild cognitive impairment $(\mathrm{aMCl})$ and Alzheimer's disease (AD). The morphometric and resting-state FC MRI analysis including 46 participants with $A D, 32$ with aMCl and 42 age-matched normal controls (NCs) were conducted. We compared the cerebellar gray matter volume and cerebellar FC with cerebral cortical regions among three groups. To investigate the relationship of cerebellar FC with cognition, we measure the correlation of significant altered FC and individual cognitive domain. No significant morphometric differences of cerebellum was observed across three groups. The patients with AD had weaker cerebral cortical FCs in bilateral Crus I and left VIllb compared to NCs, and in bilateral Crus I compared to patients with aMCl. For patients with aMCl, the weaker $\mathrm{FC}$ were found between right Crus I, left VIllb and cerebral cortical regions compared to NCs. The strength of left cerebellar FC positively correlated with specific cognitive subdomains, including memory, executive function, visuospatial function, and global cognition in AD and $\mathrm{aMCl}$. These findings demonstrated the alteration of cerebellar FC with cerebral cortical regions, and the correlation of cerebellar FC and cognitive impairment in $A D$ and $\mathrm{aMCl}$.

Keywords: Alzheimer's disease, amnestic mild cognitive impairment, cerebellum, functional connectivity, resting state fMRI

\section{INTRODUCTION}

Alzheimer's disease (AD) is the most common form of dementia, leading to a heavy burden on patients, family and society. The hallmark of the pathology of $\mathrm{AD}$ is the deposition of amyloid$\beta$ (A $\beta)$ in the cerebrum. The cerebellum has been recognized as essential only for the motor control and being free of $A \beta$ deposition (Guo et al., 2016; Jacobs et al., 2018). Therefore, for a long time, the cerebellum was considered as a "standby" in $\mathrm{AD}$, and was widely used as reference region for the calculation of standardized uptake value ratio (SUVR) in molecular imaging studies (Clark et al., 2011). 
However, increasing evidences demonstrate that the cerebellum is also associated with the regulation of cognition by way of the cerebrocerebellar circuits (Schmahmann et al., 2019). With the advancement of staining techniques, pathological studies also found the deposition of $\mathrm{A} \beta$, neurofibrillary tangles and increased microglia on cerebellar cortex in AD (Rudzinski et al., 2008; Sepulveda-Falla et al., 2014). Increasing neuroimaging studies reported the cerebellar atrophy and functional alteration in AD (Bai et al., 2011; Wang et al., 2011; Lou et al., 2015; Mascali et al., 2015; Guo et al., 2016; Jacobs et al., 2018; Olivito et al., 2020). However, till now, the studies about the changes of cerebellum in $\mathrm{AD}$ patients are still limited, and results are not consistent. This inconsistency may be due to the poor overlap of cerebellar subregions in parcellation by conventional wholebrain methods. Moreover, most previous studies focused on the relation between cerebellum and global cognitive function, the specific cognitive domain correlates with the alteration of cerebellum remains uncertain. More evidence is needed to illustrate the role of cerebellum in AD.

We hypothesized that altered cerebellar volume and functional connectivity (FC) correlated with cognitive dysfunction, and correlated with cognitive impairment in $\mathrm{AD}$ continuum. As amnestic mild cognitive impairment (aMCI) has a high incidence of conversion to AD, aMCI also provides a good model to investigate subtle change at the initial stage of the $\mathrm{AD}$ continuum (Petersen et al., 2001). Comparing the change of cerebellum among normal controls (NCs), patients with aMCI and AD, could help us to illustrate the processing of change in different cognitive status. With a cerebellum-specific spatially unbiased infratentorial template (SUIT), we performed voxel-based morphometry (VBM) analysis to compare the cerebellar volume between patients across different cognitively populations, including $\mathrm{AD}$, aMCI and NCs. In addition, we used resting-state functional MRI (rs-fMRI) to investigate the FC between cerebellum and cerebral cortical regions, and the relations between altered FC and specific cognitive domains were calculated.

\section{MATERIALS AND METHODS}

\section{Study Design and Participants}

This is a retrospective case-control study. Participants were recruited in preparation for this study from the memory clinic of China-Japan Friendship Hospital from 2014 to 2019. Participants with structural and rs-fMRI images were enrolled using the inclusion and exclusion criteria below. Patients with AD met the diagnostic criteria of probable $\mathrm{AD}$ dementia according to the new National Institute on Aging-Alzheimer's Association criteria of 2011 (McKhann et al., 2011). The inclusion criteria for AD included: (1) significant episodic memory problems reported by the patient, relative or caregiver, which was corroborated by the score of Rey Auditory Verbal Learning Test (AVLT); (2) impaired performance on general cognition test (MiniMental State Examination (MMSE) score $<24$ ) and activities of daily living (ADL); (3) medial temporal lobe atrophy on visual atrophy rating scale (Scheltens et al., 1992). Patients with aMCI participants satisfied with the Petersen's criteria and the National Institute on Aging-Alzheimer's Association criteria for MCI due to AD (van den Burg and Kingma, 1999; Petersen et al., 2014). The inclusion criteria were as follows: (1) memory complaint; (2) scoring lower than 1.5 standard deviations of the age- and education-adjusted norm on the score of AVLT; (3) normal performance on general cognition test (MMSE score $\geq 24$ ) and ADL. The NCs included family members of patients, who did not have cognitive complaints or significant decline on the neuropsychological testing, and with MMSE score $\geq 24$. The NCs were matched with $\mathrm{AD}$ and aMCI participants in gender and age.

Exclusion criteria for all participants included: (1) current or previous history of significant neurological disorder that could cause cognitive decline, including stroke, epilepsy, head trauma, intracranial mass or normal pressure hydrocephalus; (2) history of addictions or other psychiatric disorders, including schizophrenia, bipolar disorder or depression; (3) other severe medical problems, including chronic heart failure and chronic respiratory insufficiency; (4) left handed.

\section{Clinical and Neuropsychological Assessment}

All participants underwent neurological evaluation and comprehensive neuropsychological assessment. The neuropsychological assessments included general cognitive status and a series of detailed cognitive tests for specific cognitive domains, including memory, language, executive function, attention and visuospatial function (Supplementary Material 1 provide the details). The $z$ score of each cognitive domain and the composite cognitive $z$ score (average of the five individual cognitive domains) were computed based on normative data from 114 healthy control participants with similar age, education, and gender distribution (age: $69.8 \pm 6.4$; Male/Female: 47/67, education $14.8 \pm 2.8$ years, no history of neurological or psychiatric illness) in our center. Neuropsychiatric symptoms and functional impairment were assessed by caregiver-based questionnaires: Neuropsychiatric Inventory (NPI) and ADL, respectively (Cummings, 1997). The APOE genotype was determined from genotyping of isolated DNA from blood. The participants who had at least 1 APOE $\varepsilon 4$ allele were considered as APOE $\varepsilon 4$ carriers.

\section{MRI Data Acquisition and Preprocessing}

The rs-fMRI images and T1-weighted MRI images were acquired using a $3.0 \mathrm{~T}$ MR imaging system (GE Healthcare, Discovery MR750, Milwaukee, WI, United States) in the Radiology Department of China-Japan Friendship Hospital. The parameters of sagittal three-dimensional T1-weighted images with fast spoiled gradient-echo sequences (FSPGR) were as follows: echo time $(\mathrm{TE})=3.0 \mathrm{~ms}$, repetition time $(\mathrm{TR})=6.9 \mathrm{~ms}$, slice thickness $=1.0 \mathrm{~mm}, \mathrm{FOV}=256 \mathrm{~mm} \times 256 \mathrm{~mm}$, acquisition matrix $=256 \times 256$, and flip angle $=12^{\circ}$. The parameters of axial resting-state data were as follows: $\mathrm{TE}=30 \mathrm{~ms}$, $\mathrm{TR}=2,000 \mathrm{~ms}$, slice thickness $=3.0 \mathrm{~mm}, 33$ slices, field of view $(\mathrm{FOV})=240 \mathrm{~mm} \times 240 \mathrm{~mm}$, in plane matrix $=64 \times 64$, flip angle $=90^{\circ}$, and 240 phases. 
Structural three-dimensional (3-D) T1 images were first processed using the SUIT toolbox ${ }^{1}$ implemented in the Statistical Parametric Mapping software version 12 (SPM12) ${ }^{2}$ toolbox (Diedrichsen et al., 2009). Each cerebellum was separated by a Bayesian algorithm into gray matter (GM) and white matter (WM), normalized to the Montreal Neurological Institute (MNI) space using the high-resolution probability template in SUIT. The intensity of each voxel was modulated to conserve the regional differences in the total amount of GM. All the images were smoothed with a $4-\mathrm{mm}$ full-width at half-maximum (FWHM) Gaussian kernel.

The rs-fMRIs were preprocessed with the Data Processing Assistant for Resting-State fMRI (DPARSF) and the Resting-State fMRI Data Analysis Toolkit (REST). First, the first 10 volumes were discarded for the signal equilibrium and adaptation of subjects to the scanning noise. The remaining 230 volumes were corrected for timing difference and realigned to the first volume to correct for possible movement. The frame-wise displacement (FD) (Jenkinson) was calculated to evaluate the mismatch of volume-to-volume superimposed head position. The mean FD for the all the participants were $0.21 \pm 0.14 \mathrm{~mm}$. The data of 6 subjects ( $4 \mathrm{AD}$, and $2 \mathrm{NC}$ ) were excluded in this step due to excessive head motion (greater than $2.5 \mathrm{~mm}$, greater than $2.5^{\circ}$ angular rotation or mean $\mathrm{FD}>$ mean $\mathrm{FD}+2 \mathrm{SD})$. After removing the 6 subjects, the FD showed no significant different among the different groups (AD: $0.19 \pm 0.09 \mathrm{~mm}$, aMCI: $0.18 \pm 012 \mathrm{~mm}$; NC: $0.19 \pm 0.09 \mathrm{~mm}, F$-value estimated by one-way ANOVA was $0.45, p=0.64)$. To normalize the resting images, the $\mathrm{T} 1$ images were registered to their corresponding functional images and were then segmented into GM, WM, and cerebrospinal fluid tissue (CSF) probabilistic maps using a unified segmentation algorithm. Second, a GM population template was derived from the whole image data set with the DARTEL technique. Third, non-linear warping of the segmented images was then performed to match the MNI space DARTEL template. Spatial smoothing was then performed with an isotropic 4-mm FWHM Gaussian kernel. Next, linear detrending and temporal band-pass filtering $(0.01-0.1 \mathrm{~Hz})$ were applied to remove low-frequency drifts and high-frequency noise. Finally, the nuisance variables (including 6 head motion parameters and their derivatives, the WM and CSF signal, and the linear term) were regressed out.

\section{Seed ROls}

Previous studies revealed that the posterior lobe (VI, VIIb, VIII), ansiform lobe (Crus I, Crus II), and flocculonodular lobe (IX, X) of cerebellum are especially associated with cognition (Barton and Harvey, 2000; Whiting and Barton, 2003; Prevosto et al., 2010; Stoodley and Schmahmann, 2010). Therefore, these lobules were included as seed ROIs. The masks of these ROIs were extracted from the probabilistic cerebellar atlas used in SUIT (Diedrichsen et al., 2009; Figure 1). For each participant, the voxel of each seed was extracted to obtain the average seed point time series. A correlation coefficient map for each seed was produced by correlating the

${ }^{1}$ http://www.diedrichsenlab.org/imaging/suit.htm

${ }^{2}$ http://www.fil.ion.ucl.ac.uk/spm/ coefficients between the reference time series and the time series from all other brain voxels, which was then transformed to Fisher $z$-values.

\section{Statistical Analysis}

Data were analyzed using SPSS 22.0 (IBM Corp., Chicago, IL, United States). Demographic and clinical variables were checked for normality of distribution using KolmogorovSmirnov tests. Variables revealing normal distribution were compared across groups via ANOVA followed by Bonferroni post hoc tests if ANOVA was significant $(p<0.05)$. Group comparisons of NPI and ADL between AD and aMCI were performed using Student's $t$ test. Gender and ApoE4 status data were analyzed using a Chi-square test. $p<0.05$ was regarded as significant.

The resulting images were subsequently entered into a VBM analysis to perform the one-way ANOVAs to identify differences among groups in the cerebellum. Age, gender, and total intracranial volume were included as nuisance covariates. Statistical analyses were performed on smoothed GM maps within the framework of the general linear model. A one-way ANOVA model was used for assessing between group differences in regional GM cerebellar volumes. Age, sex, and years of education were included as covariates in our analysis.

ANOVA analysis was used to compare the whole-brain FC of each seed among the three different groups. The age, gender, education level, head movement parameters, and total intracranial volume were used as covariates. False discovery rate (FDR) correction was performed with a threshold of 0.05 . The $z$-values of FC were extracted to perform the post hoc $t$ test in order to identify the inter-group differences between $\mathrm{AD}$ and $\mathrm{NC}$, aMCI and NC, and aMCI and AD. Bonferroni correction was performed to adjust for the multiple testing, with a $p$ value of $<0.0167(0.05 / 3)$ considered statistically significant for these comparisons.

Due to the small sample size of individual group, we combined the patients with aMCI and $\mathrm{AD}$ together to investigate the association between all the significant different FC and the five individual cognitive subdomain. When combined aMCI and AD groups together, the $z$ scores of each cognitive subdomain did not present a normal distribution (Shapiro-Wilk test, $p<0.05$ ), therefore, the Spearman's correlation was used. The Bonferroni correction was used for multiple comparisons correction with $p<0.01(0.05 / 5)$ was considered significant.

\section{Confirmatory Analysis Based on the ADNI Dataset}

The Alzheimer's disease Neuroimaging Initiative (ADNI) database $^{3}$ was used to verify the results obtained from our data. Participants who with availability of T1-weighted MRI and rs-fMRI images were selected in the confirmatory study. The T1-weighted MRI images were performed as follows $\mathrm{TE}=3.13 \mathrm{~ms}, \mathrm{TR}=6.77 \mathrm{~ms}$, voxel size $=1 \mathrm{~mm}$ isotropic, FOV $=256 \mathrm{~mm} \times 256 \mathrm{~mm}$, acquisition matrix $=256 \times 256$. The parameters of rs-fMRI data were as follows: $\mathrm{TE}=30 \mathrm{~ms}$,

${ }^{3}$ adni.loni.usc.edu 


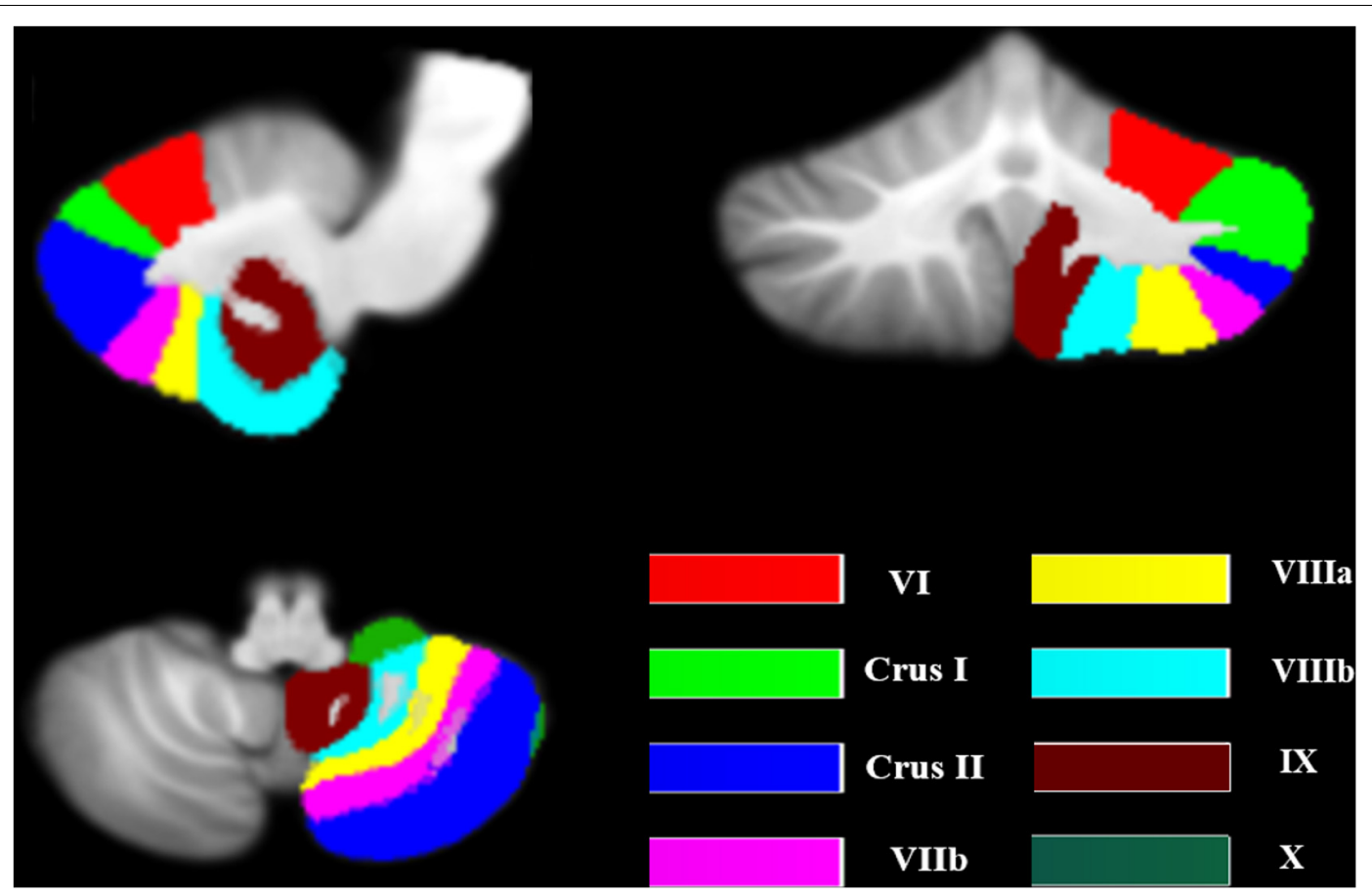

FIGURE 1 | The seeds of the cerebellum. The image was transformed into the space of the SUIT atlas and was overlapped by the seeds. The different colors show the lobular parcellation.

$\mathrm{TR}=3,000 \mathrm{~ms}, 48$ slices, voxel size $=3.3125 \mathrm{~mm}$ isotropic, $\mathrm{FOV}=256 \mathrm{~mm} \times 256 \mathrm{~mm}$, and 140 phases. The full details of imaging data ${ }^{4}$ can be found on the ADNI web site. 189 participants were recruited, however, 11 participants were excluded because of the poor quality of rs-fMRI data or excessive head motion. Finally, subjects with AD $(n=54)$, MCI $(n=57)$ and NC $(n=67)$ were selected. Details of the diagnostic criteria were from the ADNI web site ${ }^{5}$.

\section{RESULTS}

\section{Demographic and Neuropsychological Results}

From June 2014 to June 2019, we recruited 46 subjects with $\mathrm{AD}, 32$ aMCI, and $42 \mathrm{NCs}$ with the aforementioned procedures (Figure 2). Table 1 shows the clinical and neuropsychological data. No significant differences in age, gender and education level. Regarding the cognitive performance, the AD group had significantly lower scores than both the aMCI and NC groups on general cognitive test and all the cognitive subdomains except attention. aMCI subjects had significantly lower score on memory, language and executive function than NC subjects. The patients with $\mathrm{AD}$ scored significantly higher score on the ADL and NPI compared to patients with aMCI.

\footnotetext{
${ }^{4}$ http://adni.loni.usc.edu/methods/documents/mri-protocols

${ }^{5}$ http://www.adni-info.org/Scientists/AboutADNI.aspx
}

\section{Cerebellar Morphometry}

No significant group difference in volume of any cerebellar lobular was found among three groups with predefined threshold.

\section{Seed-Based FC}

Table 2 and Figure 3 illustrate the significant difference clusters in the FC for each seed among three groups. The patients with $\mathrm{AD}$ had weaker cerebral cortical FCs in bilateral Crus I, left VIIb compared to NCs. The weakened cerebellar FCs with visual cortex [Brodmann area, (BA) 18, 19), including precuneus and cuneus, were found in left VIIb and IX in AD. The left Crus I in $\mathrm{AD}$ had weaker correlations with dorsal lateral prefrontal cortex (DLPFC), inferior frontal gyrus (IFG), and anterior cingulate cortex (ACC) (BA 9, 32, 45, 46). The weakened correlations with associative visual cortex (ASC), fusiform gyrus (FG) and middle temporal gyrus (MTG) (BA19, 21, 37) were found in right Crus I. Among cerebral cortical FCs mentioned above, the weakened FCs in bilateral Crus I still showed significant compared to patients with aMCI. For patients with aMCI, the right Crus I, and Left VIIb and IX was found with significantly weaker cerebral cortical FC compared to NCs.

Cerebral cortical FC in other seeds of cerebellum was not significantly different among three groups.

\section{Cognitive Correlations of Cerebellar FC}

Cognitive correlates of the FC findings in patients with $\mathrm{AD}$ and aMCI were investigated for all reported significant cerebellar-cerebral cortical FCs after controlling for age, gender and education (Table 3 and Figure 4). For left Crus I, 


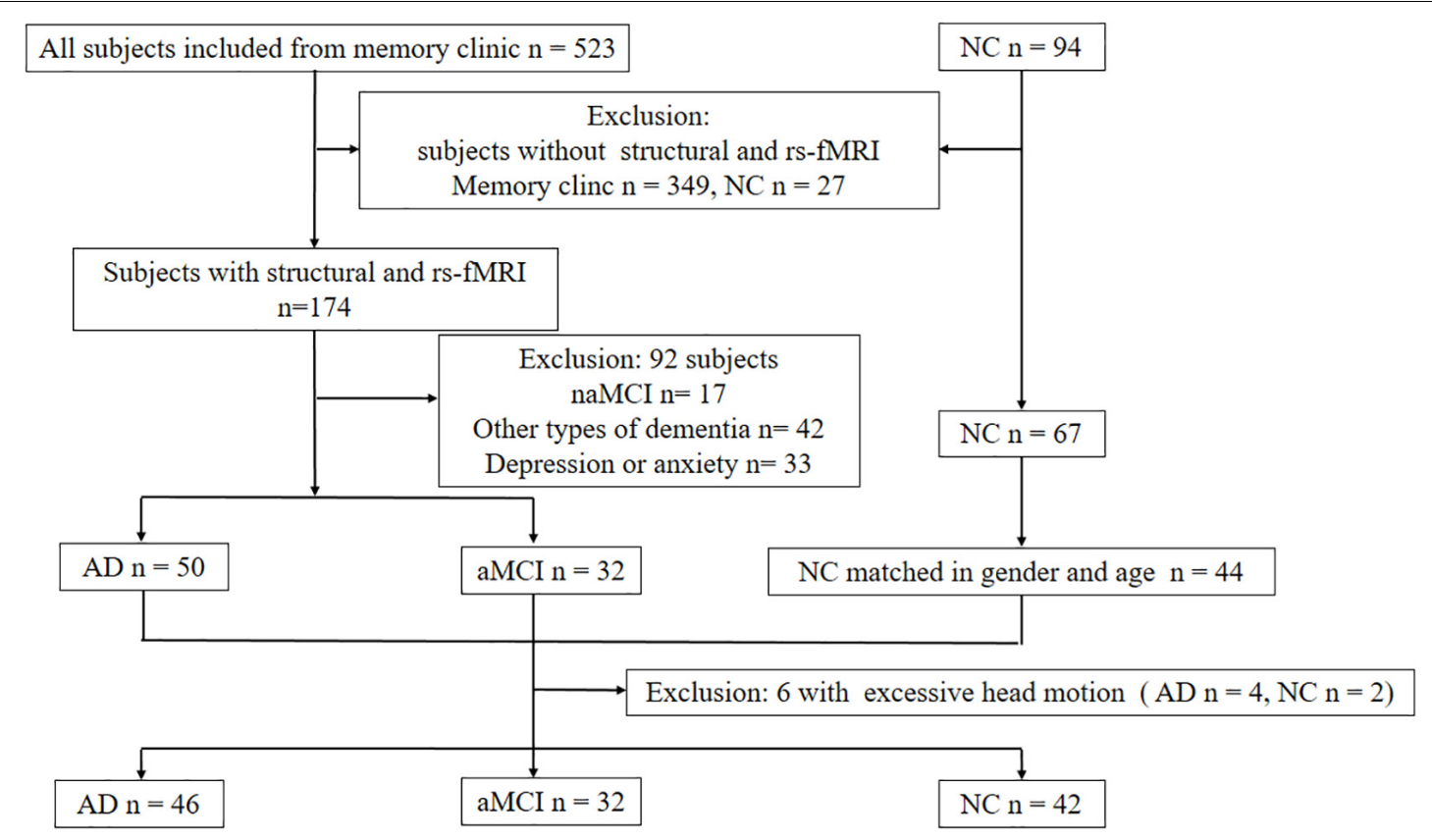

FIGURE 2 | Diagram showing the number and flow of subjects in this study. Abbreviations: NC: normal control; aMCl: amnestic mild cognitive impairment; naMCl: non-amnestic mild cognitive impairment; rs-fMRI: resting-state functional MRI.

TABLE 1 | Demographic and neuropsychological data.

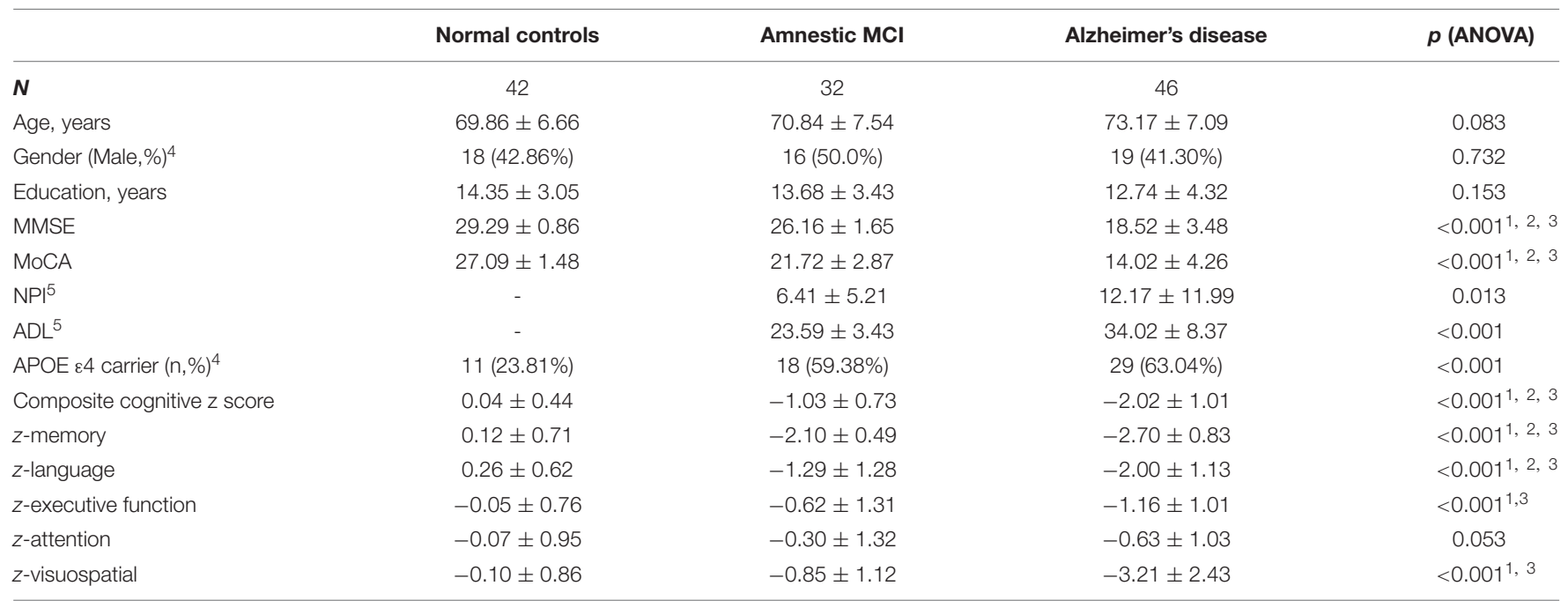

MMSE, mini-mental status examination; MoCA, Montreal Cognitive Assessment; NPI, Neuropsychiatric Inventory; ADL, Activity of Daily Living.

(1). Post hoc analysis showed significant group differences between NC and AD.

(2). Post hoc analysis showed significant group differences between NC and aMCI.

(3). Post hoc analysis showed significant group differences between $\mathrm{aMCl}$ and $\mathrm{AD}$.

(4). Values were mean \pm standard deviation. Comparisons using Chi-square test.

(5). Values were mean \pm standard deviation (sd). Comparisons using Student's t test.

the strength of FC with left DLPFC and ACC (BA 9, 32), positively correlated with executive function and visuospatial function (Figures $4 \mathbf{A}, \mathbf{B}$ ). The strength of FC between left Crus I and right DLPFC and IFG (BA 45, 46) correlated with global cognition, executive function and visuospatial function (Figures 4C-E). The FC of right Crus I with left ASC and FG correlated with memory (Figure 4F). No significant correlation with other cognitive subdomain was found. In the cerebral cortical FC with Left VII, IX, no significant correlation was found with individual cognitive domain and global cognition. The correlation for aMCI and AD subgroup is detailed in Supplementary Table 2. 
TABLE 2 | Brain regions showing significant differences during one-way ANOVA on z value of functional connectivity maps of $\mathrm{NC}$, aMCl, and AD groups.

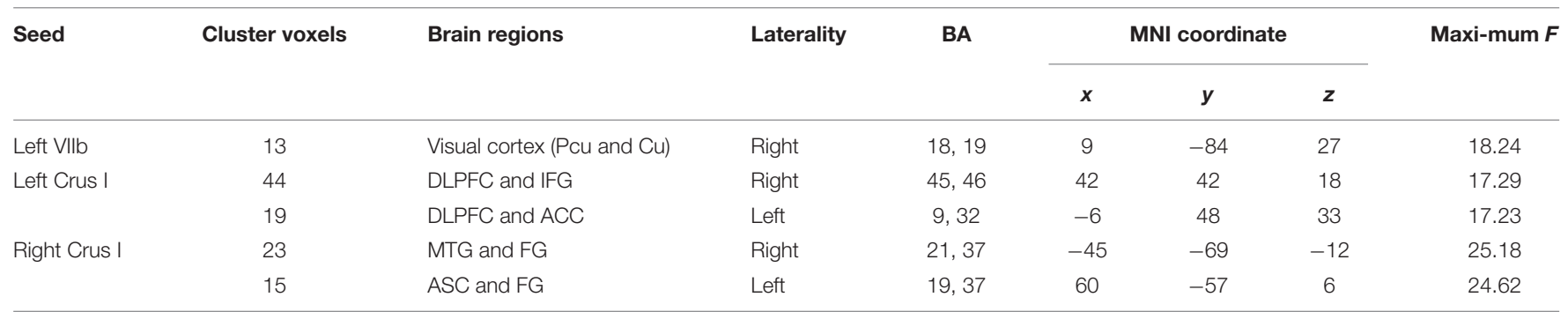

BA, Brodamann area; $P C$, precuneus; $C$, cuneus; $D L P F C$, dorsolateral prefrontal cortex; IFG, inferior frontal gyrus; $A C C$, anterior cingulate cortex; MTG, middle temporal gyrus; FG, fusiform gyrus; ASC, associative visual cortex.

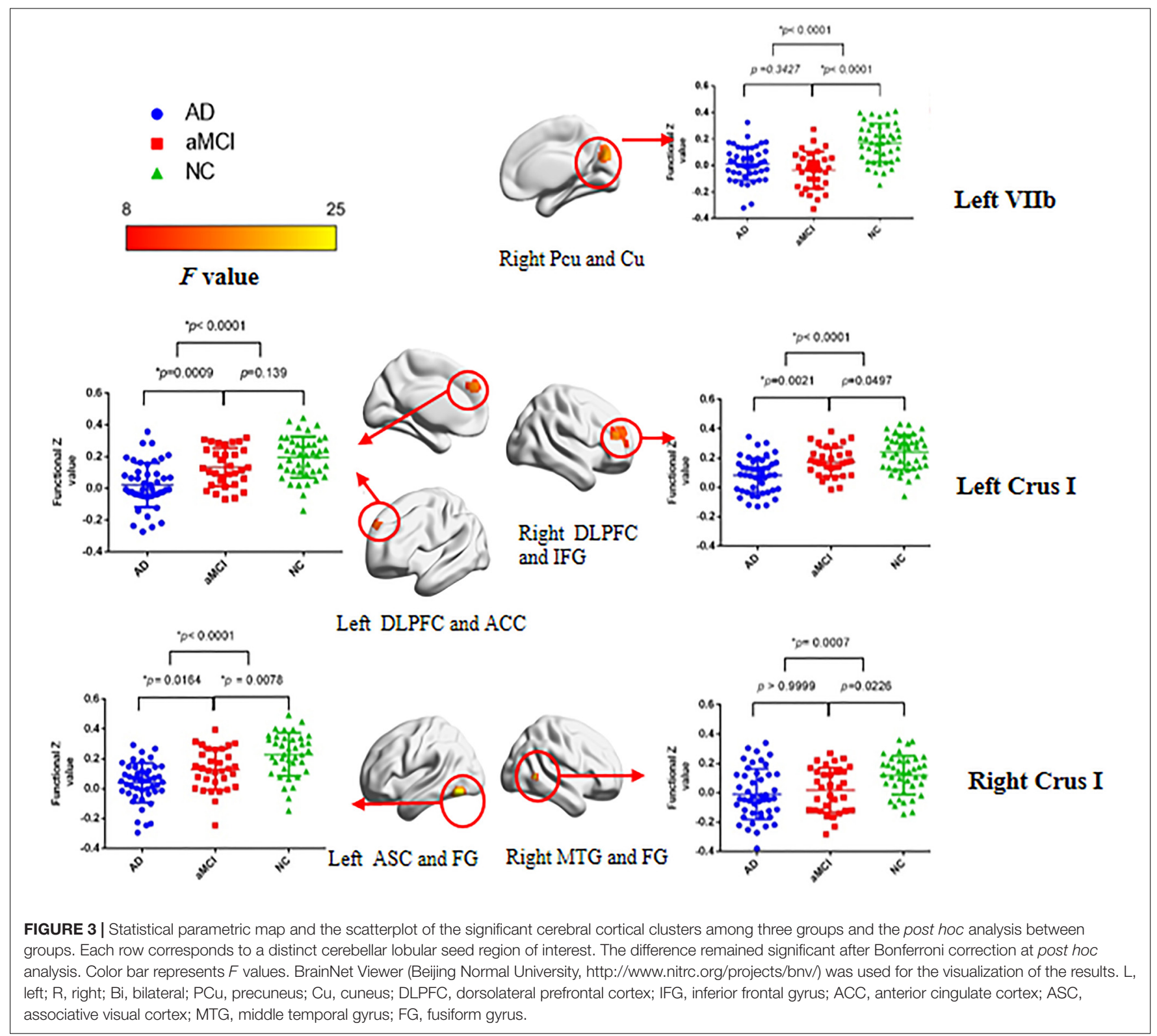


TABLE 3 | Spearman Correlation between cognition and cerebellar FC.

\begin{tabular}{|c|c|c|c|c|c|c|c|c|}
\hline BA & & & $\begin{array}{l}\text { Composite } \\
\text { z score }\end{array}$ & $\begin{array}{c}\text { Memory z } \\
\text { score }\end{array}$ & $\begin{array}{c}\text { Language } \\
\text { z score }\end{array}$ & $\begin{array}{c}\text { Executive } \\
\text { z score }\end{array}$ & $\begin{array}{l}\text { Attention } \\
\text { z score }\end{array}$ & $\begin{array}{c}\text { Visuospatial } \\
\text { z score }\end{array}$ \\
\hline \multirow[t]{2}{*}{ Left VIlb } & Right BA 18, 19 & $r$ & 0.018 & 0.010 & -0.085 & 0.057 & -0.070 & 0.062 \\
\hline & & $p$ & 0.876 & 0.930 & 0.457 & 0.619 & 0.548 & 0.594 \\
\hline \multirow[t]{4}{*}{ Left Crus I } & Left BA 9,32 & $r$ & 0.270 & 0.159 & 0.161 & $0.301^{*}$ & 0.094 & $0.327^{\star}$ \\
\hline & & $p$ & 0.017 & 0.164 & 0.159 & 0.007 & 0.415 & 0.004 \\
\hline & Right BA 45, 46 & $r$ & $0.320^{\star}$ & 0.191 & 0.252 & $0.314^{\star}$ & 0.054 & $0.387^{\star}$ \\
\hline & & $p$ & 0.004 & 0.093 & 0.026 & 0.005 & 0.644 & 0.001 \\
\hline \multirow[t]{4}{*}{ Right Crus I } & Left BA 19, 37 & $r$ & 0.204 & $0.397^{\star}$ & 0.272 & 0.241 & 0.095 & -0.030 \\
\hline & & $p$ & 0.073 & 0.000 & 0.016 & 0.034 & 0.413 & 0.795 \\
\hline & Right BA 21, 37 & $r$ & 0.138 & 0.233 & 0.143 & 0.116 & 0.144 & 0.030 \\
\hline & & $p$ & 0.230 & 0.040 & 0.213 & 0.313 & 0.213 & 0.794 \\
\hline
\end{tabular}

BA, Brodamann area. Bold and * means $p<0.05$.

\section{Results of the Confirmatory Analysis on the ADNI Dataset}

Results of the ADNI data showed similarities and differences with those obtained from our sample.

In the VBM analysis, compared with the $\mathrm{NC}$ group, the $\mathrm{AD}$ and aMCI groups had significant gray matter volume reduction in the left Crus I/II, and there is no difference between the $\mathrm{AD}$ and aMCI group (NC > AD: $p<0.001$;C $>$ aMCI: $p<0.001$; aMCI $>$ AD: $p=0.059)$. Supplementary Table 3 and Supplementary Figure 1 show the details.

The seed-based FC analysis based on ADNI database showed that the cortical FC in left IX, left Crus I and bilateral Crus II are involved in aMCI group, with a later involvement of the right $\mathrm{X}$ lobe in $\mathrm{AD}$ group only. Similar to our sample, the weakened cortical cerebellar FCs involved left DLPFC, FG, bilateral precuneus and cuneus. Details are illustrated in Supplementary Table 4 and Supplementary Figures 2-5.

\section{DISCUSSION}

This case-control study investigated the cerebellar anatomic and functional changes across three different cognitive status, including the $\mathrm{NC}$, aMCI, and $\mathrm{AD}$ group. The strength of cerebellar FC with cerebral cortical areas were different among three groups, and it correlated with cognitive function in $\mathrm{AD}$ and aMCI. The results from ADNI cohort partially confirmed these findings.

The weakened FC was found between the left VIIb and contralateral precuneus and cuneus in AD. Precuneus is one of the core regions of default mode network (DMN) (Buckner et al., 2008). $A \beta$ accumulation preferentially starts in several of the core regions of the $\mathrm{DMN}$, including the precuneus at the early stage of AD (Palmqvist et al., 2017). From the perspective of clinical symptoms, the DMN has been found to be related to episodic memory in AD (Buckner et al., 2008; Sperling et al., 2009). Failure to detect the correlation with the cognitive performance, especially with the memory, could be due to the restricted range of this dependent variable in our aMCI cohort.

Compared to NC, the aMCI and $\mathrm{AD}$ group showed weaker FC of the left Crus I correlated with frontal lobe (bilateral
DLPFC, left ACC and right IFG), while right Crus I with occipital and temporal lobule (bilateral FG, left ASC and right MTG). The role of Crus I in working memory, planning and organization have been highlighted by functional imaging studies (Buckner, 2013). In addition, the role of DLPFC in executive function had been clearly established. This is consistent with our result that the FC between left Crus I and bilateral DLPFC correlated with execution. Previous fMRI also demonstrated the crossed cerebro-cerebellar projections, language is heavily right lateralized and visuospatial function left lateralized. Interestingly, in this study, we found similar lateralization in Crus I, as the FC of left Crus I connected with the execution and visuospatial function, and right Crus I connected with the memory and language.

Crossed cerebellar diaschisis (CCD) is the remote effect of supratentorial dysfunction in the unilateral hemisphere inducing contralateral cerebellar hypometabolism (Lin, 1997), which could explain the weakened FC between cerebellum and cerebrum. The mechanism of CCD include the involvement of cortico-pontocerebellar and the cerebello-thalamo-cortical circuits (Dum and Strick, 2003; Di Lorenzo et al., 2013). Using diffuse tension imaging (DTI), Sofia Toniolo et al. provide the evidence of the impairment of microstructural fiber integrity of cerebellum WM tracts in AD (Toniolo et al., 2020). However, whether the focal change in the cerebellum is a form of Wallerian degeneration or the result of accumulation of $\mathrm{AD}$ pathological substrates in the cerebellum itself is still unknown. In this study, we did not find morphometric difference in any of the observed cerebral cortical regions across the three groups, also implicating altered FC could be due to the dysfunction of neurotransmitter or network connection, instead of being secondary to the atrophy.

In this study, we did not find significant differences in volumes of any cerebellar lobular in $\mathrm{AD}$ or aMCI group from the data of our center, though the results based on ADNI database showed the GM volume loss in left Crus I/II. The smaller sample size and the younger age of our cohort might account for the discrepancy. Using the SUIT template for cerebellar VBM parcellation, which is the same method as our study, Sofia Toniolo et al. also reported a progression of cerebellar GM volume loss throughout a continuous spectrum from aMCI stage to AD stage (Toniolo et al., 2018). 


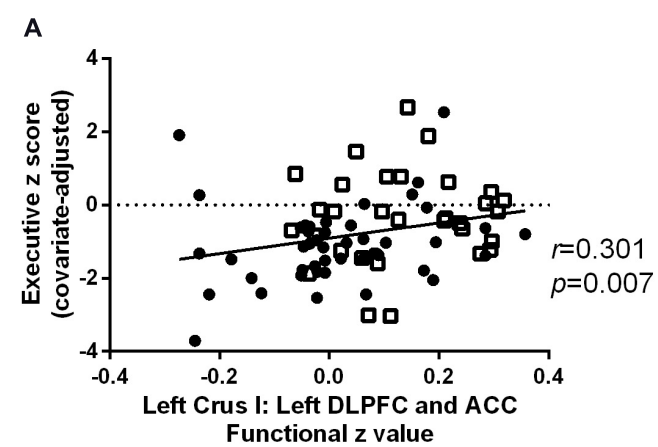

C

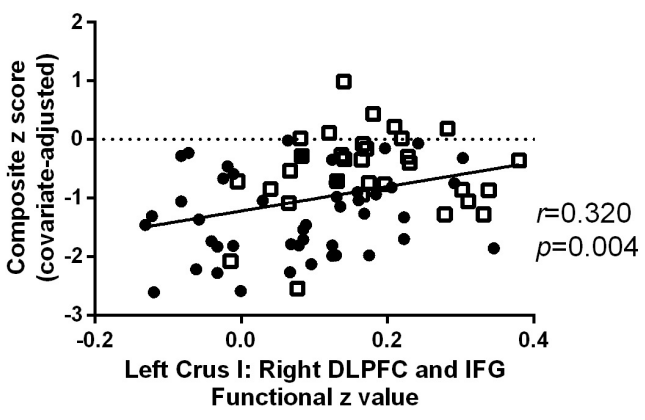

E

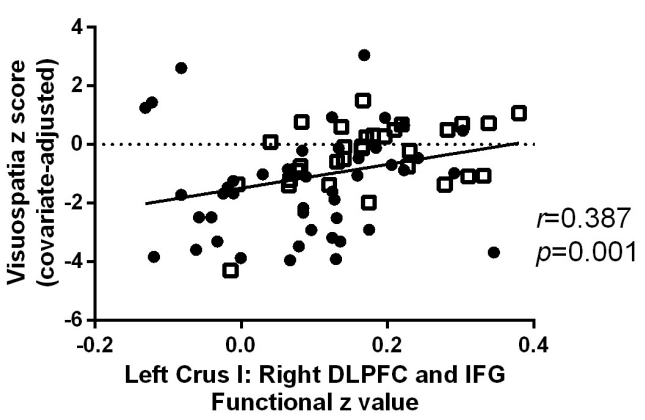

B

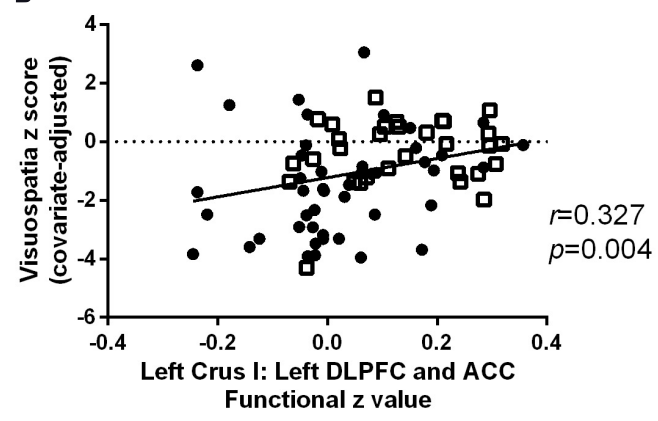

D

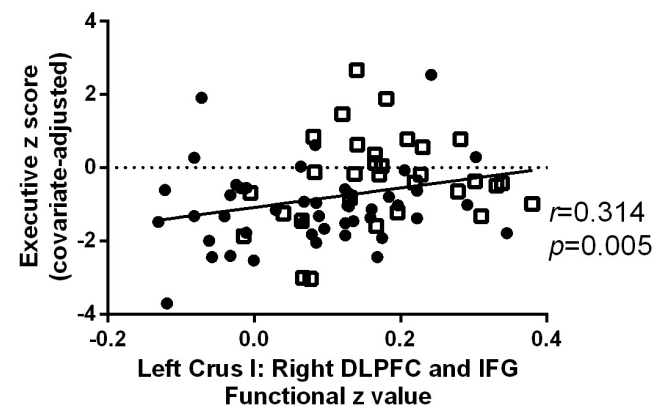

$\mathbf{F}$

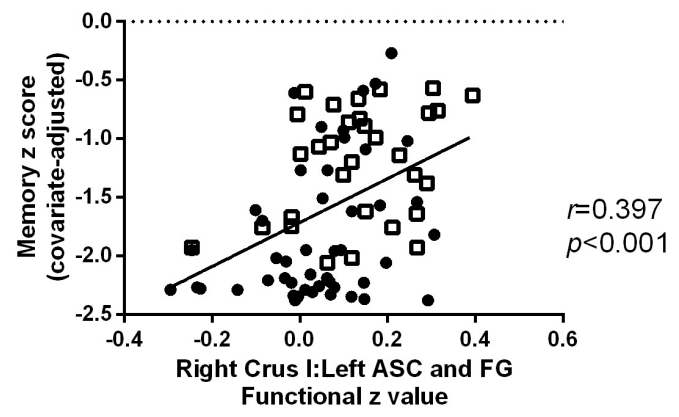

FIGURE 4 | Scatter plots for the significant cognitive-functional connectivity (FC) correlations in Alzheimer's disease (AD) (circles) and amnestic mild cognitive impairment (aMCI) (squares). The strength of FC with left dorsolateral prefrontal cortex (DLPFC) and inferior frontal gyrus (IFG) positively correlated with (A) executive and (B) visuospatial function. The FC of left Crus I and correlated with (C) global cognition, (D) executive function, and (E) visuospatial function. The FC of right Crus I with left associative visual cortex (ASC) and fusiform gyrus (FG) positively correlated with memory (F).

To investigate the mechanism of cerebellar involvement in $\mathrm{AD}$ and aMCI is important for the diagnosis and treatment. Recent strategies of diagnosis and treatment for $\mathrm{AD}$ continuum are based on identification and quantification of the pathological biomarkers. Molecular neuroimaging study with selective radioligands, including $A \beta$ and phosphorylated tau, is an important method for the quantification of these biomarkers (Jack et al., 2018). The most used parameter is the SUVR between a target region and a reference region (Fleisher et al., 2011). The selection of reference region directly affects the value of SUVR, which therefore is important for the diagnosis. The cerebellum has been the most widely used reference region in AD (Clark et al., 2011; Maass et al., 2017; Stern et al., 2019). However, if the cerebellum is involved in the pathogenesis of $\mathrm{AD}$ continuum, it may not be an optimal choice for the reference region. Furthermore, using the repetitive transcranial magnetic stimulation (rTMS) of cerebellum, Di Lorenzo et al. (2020) revealed the impairment of cerebellar-cortical plasticity by showing the long term potentiation (LTP) was impaired in AD patients. For the treatment, as the cerebellum is easily accessible with non-invasive stimulation tools, it may be used as a novel target for neuromodulation in $\mathrm{AD}$ in the future. 
There are some limitations to this study. First, as a retrospective case-control study, the identification of the aMCI and $\mathrm{AD}$ groups was not based on pathological evidence, such as $\mathrm{A} \beta \mathrm{PET}$, which is still expensive for some patients in China. However, in this study, the prevalence of APOE $\varepsilon 4$ carriers in $\mathrm{AD}$ and aMCI was 61.70 and $59.38 \%$, respectively, which is similar to that of a previous study with large sample of $A \beta$ biomarker positive individuals (66\% in $\mathrm{AD}$ and $64 \%$ in $\mathrm{MCI}$ ) (Mattsson et al., 2018). Second, though we included the aMCI group as the prodromal stage of $\mathrm{AD}$, this was still a crosssectional study. In the future, longitudinal studies are needed to investigate the dynamic changes in the cerebellum throughout disease progression.

\section{CONCLUSION}

In conclusion, these findings suggest the functional changes of the cerebellum indicating the critical role cerebellum in the cognitive impairment in aMCI and AD. This was important because using the cerebellum as the reference region for ligand neuroimaging studies could bring the possible biased results.

\section{DATA AVAILABILITY STATEMENT}

The datasets presented in this study can be found in online repositories. The names of the repository/repositories and accession number(s) can be found below: http://dx.doi.org/10. 17632/tc7xmjbmfw.3.

\section{ETHICS STATEMENT}

The studies involving human participants were reviewed and approved by Research Ethics Committees of China-Japan

\section{REFERENCES}

Bai, F., Liao, W., Watson, D. R., Shi, Y., Yuan, Y., Cohen, A. D., et al. (2011). Mapping the altered patterns of cerebellar resting-state function in longitudinal amnestic mild cognitive impairment patients. J. Alzheimer's Dis. 23, 87-99. doi: 10.3233/jad-2010-101533

Barton, R. A., and Harvey, P. H. (2000). Mosaic evolution of brain structure in mammals. Nature 405, 1055-1058. doi: 10.1038/35016580

Buckner, R. L. (2013). The cerebellum and cognitive function: 25 years of insight from anatomy and neuroimaging. Neuron $80,807-815$. doi: 10.1016/j.neuron. 2013.10.044

Buckner, R. L., Andrews-Hanna, J. R., and Schacter, D. L. (2008). The brain's default network: anatomy, function, and relevance to disease. Ann. N. Y. Acad. Sci. 1124, 1-38. doi: 10.1196/annals.1440.011

Clark, C. M., Schneider, J. A., Bedell, B. J., Beach, T. G., Bilker, W. B., Mintun, M. A., et al. (2011). Use of florbetapir-PET for imaging beta-amyloid pathology. JAMA 305, 275-283. doi: 10.1001/jama.2010.2008

Cummings, J. L. (1997). Changes in neuropsychiatric symptoms as outcome measures in clinical trials with cholinergic therapies for Alzheimer disease. Alzheimer Dis. Assoc. Disord. 11(Suppl. 4), S1-S9.

Di Lorenzo, F., Bonnì, S., Picazio, S., Motta, C., Caltagirone, C., Martorana, A., et al. (2020). Effects of cerebellar theta burst stimulation on contralateral motor cortex excitability in patients with Alzheimer's disease. Brain Topogr. 33, 613-617. doi: 10.1007/s10548-020-00781-6
Friendship Hospital. The patients/participants provided their written informed consent to participate in this study.

\section{AUTHOR CONTRIBUTIONS}

DP: conceptualization. ZZ, WS, and LW: methodology. ZZ, RZ, SZ, LW, and XD: formal analysis and investigation. ZZ: writing original draft preparation. RZ and DP: writing - review and editing. DP: funding acquisition and supervision. All authors read and approved the final manuscript.

\section{FUNDING}

This work was supported by the National Natural Science Foundation of China (Grant No. 81571657).

\section{ACKNOWLEDGMENTS}

This manuscript has been released as a pre-print at Research Square (https://doi.org/10.21203/rs.3.rs-55425/v1+). We thank the MRI technologists and operators for their assistance, and also thank all the participants for their dedication, time, and effort to this study.

\section{SUPPLEMENTARY MATERIAL}

The Supplementary Material for this article can be found online at: https://www.frontiersin.org/articles/10.3389/fnsys. 2021.596221/full\#supplementary-material

Di Lorenzo, F., Martorana, A., Ponzo, V., Bonnì, S., D’Angelo, E., Caltagirone, C., et al. (2013). Cerebellar theta burst stimulation modulates short latency afferent inhibition in Alzheimer's disease patients. Front. Aging Neurosci. 5:2.

Diedrichsen, J., Balsters, J. H., Flavell, J., Cussans, E., and Ramnani, N. (2009). A probabilistic MR atlas of the human cerebellum. NeuroImage 46, 39-46. doi: 10.1016/j.neuroimage.2009.01.045

Dum, R. P., and Strick, P. L. (2003). An unfolded map of the cerebellar dentate nucleus and its projections to the cerebral cortex. J. Neurophysiol. 89, 634-639. doi: 10.1152/jn.00626.2002

Fleisher, A. S., Chen, K., Liu, X., Roontiva, A., Thiyyagura, P., Ayutyanont, N., et al. (2011). Using positron emission tomography and florbetapir F18 to image cortical amyloid in patients with mild cognitive impairment or dementia due to Alzheimer disease. Arch. Neurol. 68, 1404-1411. doi: 10.1001/archneurol.2011. 150

Guo, C. C., Tan, R., Hodges, J. R., Hu, X., Sami, S., and Hornberger, M. (2016). Network-selective vulnerability of the human cerebellum to Alzheimer's disease and frontotemporal dementia. Brain J. Neurol. 139(Pt 5), 1527-1538. doi: 10.1093/brain/aww003

Jack, C. R. Jr., Bennett, D. A., Blennow, K., Carrillo, M. C., Dunn, B., Haeberlein, S. B., et al. (2018). NIA-AA Research framework: toward a biological definition of Alzheimer's disease. Alzheimer's Dement. 14, 535-562. doi: 10.1016/j.jalz. 2018.02.018

Jacobs, H. I. L., Hopkins, D. A., Mayrhofer, H. C., Bruner, E., van Leeuwen, F. W., Raaijmakers, W., et al. (2018). The cerebellum in Alzheimer's disease: evaluating 
its role in cognitive decline. Brain J. Neurol. 141, 37-47. doi: 10.1093/brain/ awx194

Lin, W. Y. (1997). Crossed cerebellar diaschisis: related to lesion location or disease duration? J. Nuclear Med. 38:2006.

Lou, W., Shi, L., Wang, D., Tam, C. W., Chu, W. C., Mok, V. C., et al. (2015). Decreased activity with increased background network efficiency in amnestic MCI during a visuospatial working memory task. Hum. Brain Mapp. 36, 3387-3403. doi: 10.1002/hbm.22851

Maass, A., Landau, S., Baker, S. L., Horng, A., Lockhart, S. N., La Joie, R., et al. (2017). Comparison of multiple tau-PET measures as biomarkers in aging and Alzheimer's disease. NeuroImage 157, 448-463. doi: 10.1016/j.neuroimage. 2017.05.058

Mascali, D., DiNuzzo, M., Gili, T., Moraschi, M., Fratini, M., Maraviglia, B., et al. (2015). Intrinsic patterns of coupling between correlation and amplitude of lowfrequency fMRI fluctuations are disrupted in degenerative dementia mainly due to functional disconnection. PLoS One 10:e0120988. doi: 10.1371/journal.pone. 0120988

Mattsson, N., Groot, C., Jansen, W. J., Landau, S. M., Villemagne, V. L., Engelborghs, S., et al. (2018). Prevalence of the apolipoprotein E epsilon4 allele in amyloid beta positive subjects across the spectrum of Alzheimer's disease. Alzheimer's Dement. 14, 913-924.

McKhann, G. M., Knopman, D. S., Chertkow, H., Hyman, B. T., Jack, C. R., Kawas, C. H., et al. (2011). The diagnosis of dementia due to Alzheimer's disease: recommendations from the national institute on aging-alzheimer's association workgroups on diagnostic guidelines for Alzheimer's disease. Alzheimer's Dement. 7, 263-269.

Olivito, G., Serra, L., Marra, C., Di Domenico, C., Caltagirone, C., Toniolo, S., et al. (2020). Cerebellar dentate nucleus functional connectivity with cerebral cortex in Alzheimer's disease and memory: a seed-based approach. Neurobiol. Aging 89, 32-40. doi: 10.1016/j.neurobiolaging.2019.10.026

Palmqvist, S., Scholl, M., Strandberg, O., Mattsson, N., Stomrud, E., Zetterberg, H., et al. (2017). Earliest accumulation of beta-amyloid occurs within the defaultmode network and concurrently affects brain connectivity. Nat. Commun. 8:1214.

Petersen, R. C., Caracciolo, B., Brayne, C., Gauthier, S., Jelic, V., and Fratiglioni, L. (2014). Mild cognitive impairment: a concept in evolution. J. Intern. Med. 275, 214-228.

Petersen, R. C., Stevens, J. C., Ganguli, M., Tangalos, E. G., Cummings, J. L., and DeKosky, S. T. (2001). Practice parameter: early detection of dementia: mild cognitive impairment (an evidence-based review). report of the quality standards subcommittee of the american academy of neurology. Neurology 56, 1133-1142. doi: 10.1212/wnl.56.9.1133

Prevosto, V., Graf, W., and Ugolini, G. (2010). Cerebellar inputs to intraparietal cortex areas LIP and MIP: functional frameworks for adaptive control of eye movements, reaching, and arm/eye/head movement coordination. Cereb. Cortex 20, 214-228. doi: 10.1093/cercor/bhp091

Rudzinski, L. A., Fletcher, R. M., Dickson, D. W., Crook, R., Hutton, M. L., Adamson, J., et al. (2008). Early onset familial Alzheimer disease with spastic paraparesis, dysarthria, and seizures and N135S mutation in PSEN1. Alzheimer Dis. Assoc. Disord. 22, 299-307. doi: 10.1097/wad.0b013e318173 2399
Scheltens, P., Leys, D., Barkhof, F., Huglo, D., Weinstein, H. C., Vermersch, P., et al. (1992). Atrophy of medial temporal lobes on MRI in "probable" Alzheimer's disease and normal ageing: diagnostic value and neuropsychological correlates. J. Neurol. Neurosurg. Psychiatry 55, 967-972. doi: 10.1136/jnnp.55.10.967

Schmahmann, J. D., Guell, X., Stoodley, C. J., and Halko, M. A. (2019). The theory and neuroscience of cerebellar cognition. Annu. Rev. Neurosci. 42, 337-364. doi: 10.1146/annurev-neuro-070918-050258

Sepulveda-Falla, D., Barrera-Ocampo, A., Hagel, C., Korwitz, A., Vinueza-Veloz, M. F., Zhou, K., et al. (2014). Familial Alzheimer's disease-associated presenilin1 alters cerebellar activity and calcium homeostasis. J. Clin. Invest. 124, 15521567. doi: $10.1172 /$ jci66407

Sperling, R. A., Laviolette, P. S., O’Keefe, K., O’Brien, J., Rentz, D. M., Pihlajamaki, M., et al. (2009). Amyloid deposition is associated with impaired default network function in older persons without dementia. Neuron 63, 178-188. doi: 10.1016/j.neuron.2009.07.003

Stern, R. A., Adler, C. H., Chen, K., Navitsky, M., Luo, J., Dodick, D. W., et al. (2019). Tau positron-emission tomography in former national football league players. N. Engl. J. Med. 380, 1716-1725. doi: 10.1056/nejmoa1900757

Stoodley, C. J., and Schmahmann, J. D. (2010). Evidence for topographic organization in the cerebellum of motor control versus cognitive and affective processing. Cortex 46, 831-844. doi: 10.1016/j.cortex.2009.11.008

Toniolo, S., Serra, L., Olivito, G., Caltagirone, C., Mercuri, N. B., Marra, C., et al. (2020). Cerebellar white matter disruption in alzheimer's disease patients: a diffusion tensor imaging study. J. Alzheimer's Dis. 74, 615-624. doi: 10.3233/ jad-191125

Toniolo, S., Serra, L., Olivito, G., Marra, C., Bozzali, M., and Cercignani, M. (2018). Patterns of cerebellar gray matter atrophy across Alzheimer's disease progression. Front. Cell. Neurosci. 12:430.

van den Burg, W., and Kingma, A. (1999). Performance of 225 dutch school children on Rey's auditory verbal learning test (AVLT): parallel test-retest reliabilities with an interval of 3 months and normative data. Arch. Clin. Neuropsychol. 14, 545-559. doi: 10.1093/arclin/14.6.545

Wang, Z., Yan, C., Zhao, C., Qi, Z., Zhou, W., Lu, J., et al. (2011). Spatial patterns of intrinsic brain activity in mild cognitive impairment and Alzheimer's disease: a resting-state functional MRI study. Hum. Brain Mapp. 32, 1720-1740. doi: 10.1002/hbm. 21140

Whiting, B. A., and Barton, R. A. (2003). The evolution of the cortico-cerebellar complex in primates: anatomical connections predict patterns of correlated evolution. J. Hum. Evol. 44, 3-10. doi: 10.1016/s0047-2484(02)00162-8

Conflict of Interest: The authors declare that the research was conducted in the absence of any commercial or financial relationships that could be construed as a potential conflict of interest.

Copyright (c) 2021 Zhou, Zhu, Shao, Zhang, Wang, Ding and Peng. This is an openaccess article distributed under the terms of the Creative Commons Attribution License (CC BY). The use, distribution or reproduction in other forums is permitted, provided the original author(s) and the copyright owner(s) are credited and that the original publication in this journal is cited, in accordance with accepted academic practice. No use, distribution or reproduction is permitted which does not comply with these terms. 